\section{Validade dimensional do instrumento de qualidade de vida WHOQOL-BREF aplicado a trabalhadores de saúde}

\author{
Dimensional validity of WHOQOL-BREF in health \\ workers
}

\author{
Validez dimensional del instrumento de calidad \\ de vida WHOQOL-BREF aplicado a trabajadores \\ de salud
}

Marcelle Maria Lobo Dinis Castro 1,2 Yara Hahr Marques Hökerberg 1 Sonia Regina Lambert Passos 1

\footnotetext{
1 Instituto de Pesquisa Clínica Evandro Chagas, Fundação Oswaldo Cruz, Rio de Janeiro, Brasil. 2 Escola Nacional de Saúde Pública Sergio Arouca, Fundação Oswaldo Cruz, Rio de Janeiro, Brasil.

Correspondência M. M. L. D. Castro Instituto de Pesquisa Clínica Evandro Chagas, Fundação Oswaldo Cruz.

Av. Brasil 4365, Rio de Janeiro, RJ 21040-900, Brasil. lobodinis@gmail.com
}

\begin{abstract}
This study's objective was to revisit the internal consistency and convergent and discriminant factorial validity of the Brazilian version of WHOQOL-BREF, in a cross-sectional study of 386 health workers. Confirmatory factor analysis was used to test WHOQOL-BREF's structure (24 and 26 items) and the model suggested by exploratory factor analysis. Internal consistency was measured through composite reliability; convergent and discriminant validity by average variance extracted and factor correlations. The best-fit model, suggested by exploratory factor analysis (26 items), had 6 factors: 4 theoretically proposed (general, psychological, social relationships, and environment) and 2 obtained by subdividing the physical domain. Items "energy" and "security" (from physical and environment domains) were relocated to the psychological domain. Composite reliability was good ( $>0.70$ ), except for the general factor. Convergent and discriminant validity were adequate for social relationships and physical 2. Controversies on WHOQOL-BREF's dimensionality persist, particularly in the physical domain.
\end{abstract}

Validation Studies; Health Personnel; Quality of Life; Working Environment

\section{Resumo}

O objetivo foi reavaliar a estrutura dimensional da versão brasileira do WHOQOL-BREF, consistência interna e validade fatorial convergente $e$ discriminante. Estudo seccional em 386 trabalhadores de saúde. A análise fatorial confirmatória testou a estrutura do WHOQOL-BREF (24 e 26 itens) e a sugerida pela análise fatorial exploratória. Consistência interna foi aferida via confiabilidade composta; validade convergente e discriminante, pela variância média extraída e correlação entre fatores. O modelo de melhor ajuste foi o sugerido pela análise fatorial exploratória (26 itens) com seis fatores: quatro propostos teoricamente (geral, psicológico, relações sociais e meio ambiente) e dois pela subdivisão do domínio físico. Os itens "energia" $e$ "segurança" (respectivamente, do físico e meio ambiente) foram remanejados para o psicológico. A confiabilidade composta foi boa (>0,70), à exceção do fator geral. Validade convergente e discriminante foram adequadas para relações sociais e físico 2. Persistem controvérsias sobre a dimensionalidade do WHOQOL-BREF, particularmente sobre o domínio físico.

Estudos de Validação; Pessoal de Saúde; Qualidade de Vida; Ambiente de Trabalho 


\section{Introdução}

A qualidade de vida é considerada um construto complexo bastante utilizado em avaliação de saúde por englobar, além da saúde física, aspectos sociais, culturais, ambientais e psicológi$\cos 1,2,3$.

Para enfrentar a problemática ausência de conceito de qualidade de vida, do desenvolvimento de instrumentos com foco nos aspectos estritamente da saúde (SF-36 Health Survey, Sickness Impact Profile) e das frágeis tentativas de adaptação dos instrumentos por ser a maioria em língua inglesa e cultura local, a Organização Mundial da Saúde (OMS) desenvolveu um instrumento de qualidade de vida (WHOQOL-100) que avalia a "percepção do indivíduo de sua posição na vida, no contexto de sua cultura e do sistema de valores em que vive e em relação as suas expectativas, seus padrões e suas preocupações" 2 (p. 1405). Existem dois instrumentos do WHOQOL: o WHOQOL-100, com 100 itens distribuídos em seis domínios - físico, psicológico, meio ambiente, nível de independência, relações sociais e espiritualidade; e o WHOQOL-BREF (Tabela 1), com 26 itens distribuídos em quatro domínios - físico, psicológico, relações sociais e meio ambiente e uma faceta geral: "qualidade de vida" e "saúde". A proposta teórica do grupo WHOQOL considera a faceta geral como um fator do construto qualidade de vida ${ }^{4}$, entretanto, apenas dois 5,6 entre 18 estudos consideraram estes dois itens fazendo parte da estrutura dimensional do WHOQOL-BREF (Tabela 2).

No Brasil, o WHOQOL-BREF tem sido utilizado para aferir o desfecho em estudos de associação, em amostras de estudantes 7, trabalhadores 8,9, em pacientes com doenças clínicas crônicas e psiquiátricas 10,11,12,13,14 e em idosos 15,16.

Entre as suas propriedades psicométricas, a consistência interna do instrumento WHOQOLBREF variou de 0,90 17 a 0,93 18 para a escala global (26 itens); e de 0,45 para o domínio relações sociais 6 a 0,89 para o domínio físico ${ }^{18}$. A confiabilidade teste-reteste oscilou entre 0,66 (físico) 19 a 0,91 (psicológico) 20.

Quanto a sua dimensionalidade, nove estudos utilizaram a abordagem confirmatória para avaliar a estrutura dimensional de 24 itens distribuídos em quatro domínios, excluindo os dois itens da faceta geral ("qualidade de vida" e "saúde") 17,18,19,21,22,23,24,25,26,27. Porém, pelo menos um índice de ajuste não foi adequado - comparative fit index $(\mathrm{CFI})<0,90$ 17,18,21,22,23,25,27 e root mean square error of approximation (RMSEA) > $0,0826,27$, ou o modelo final indicou seis 6,22 ao invés de quatro fatores (Tabela 2). Análise fatorial exploratória resultou em modelos com quatro e cinco fatores ou itens agrupando-se em fatores diferentes dos sugeridos pelo grupo WHOQOL 5,20,28,29,30,31 (Tabela 2).

Até o momento, o único estudo brasileiro sobre análise fatorial confirmatória do WHOQOLBREF foi realizado em idosos confirmando a estrutura de quatro dimensões propostas originalmente 21. A maioria dos estudos de análise fatorial confirmatória utilizou o método de máxima verossimilhança que pressupõe grandes tamanhos amostrais e distribuição normal multivariada das variáveis indicadoras contínuas 18,21,23,32 . Esta reavaliação é, também, importante porque não foram identificados estudos sobre a dimensionalidade deste instrumento em trabalhadores de instituição de saúde - médicos, enfermeiros, psicólogos, assistentes sociais, profissionais que estão em contato direto com o sofrimento físico e psíquico de pacientes e nos quais estudos sobre qualidade de vida têm sido demandados, mas que constitui uma população diferente daquela em que o instrumento WHOQOL-BREF foi validado no Brasil (população geral e doentes) 33.

O objetivo do presente estudo é reavaliar a estrutura dimensional da versão brasileira do WHOQOL-BREF quando aplicada a trabalhadores de instituição de pesquisa em saúde, utilizando a abordagem confirmatória. Pretende, ainda, avaliar o papel dos dois itens da faceta geral e a validade fatorial convergente e discriminante, por meio da comparação da correlação entre os fatores com a variância média extraída, contribuindo assim para o entendimento sobre o conjunto de itens que melhor descreve o construto qualidade de vida.

\section{Método}

Os dados foram provenientes de estudo de delineamento seccional (2004-2005), cujo objetivo principal foi avaliar o perfil de saúde do censo de trabalhadores de uma instituição de saúde do Rio de Janeiro. A população fonte totalizava 497 profissionais, dos quais 50 não preenchiam critérios de inclusão por vínculo empregatício precário. Dos 447 elegíveis, constituíram perda neste estudo 61 trabalhadores: 25 que não concordaram em participar e 36 que não preencheram pelo menos uma questão do WHOQOL -BREF. Foram incluídos 386 trabalhadores. As 26 perguntas do WHOQOL-BREF avaliam os aspectos geral, físico, psicológico, sociais e ambientais do indivíduo nas últimas duas semanas. A pontuação de cada item é do tipo Likert (1 a 5) disposta de modo que os maiores valores estão relacionados a uma melhor qualidade de vida, à exceção dos itens q3 “dor física”, q4 “tratamento” 
Tabela 1

Versão brasileira do WHOQOL-BREF *

\begin{tabular}{|c|c|c|c|}
\hline Domínios & Itens & Perguntas ("Nas duas últimas semanas...") & $\begin{array}{l}\text { Opções de respostas } \\
\qquad(\mathrm{n}=5) \text { ** }\end{array}$ \\
\hline \multirow[t]{2}{*}{ Geral } & q1. qualidade de vida & Como você avaliaria sua qualidade de vida? & Muito ruim a Muito boa \\
\hline & q2. saúde & O quanto você está satisfeito(a) com a sua saúde? & $\begin{array}{l}\text { Muito insatisfeito a } \\
\text { Muito satisfeito }\end{array}$ \\
\hline \multirow[t]{7}{*}{ Físico } & q3. dor física & $\begin{array}{l}\text { Em que medida você sente alguma dor física que o impede de fazer o que você } \\
\text { precisa? }\end{array}$ & Nada a Extremamente \\
\hline & q4. tratamento & O quanto você precisa de algum tratamento médico para levar sua vida diária? & Nada a Extremamente \\
\hline & q10. energia & Você tem energia suficiente para seu dia a dia? & Nada a Completamente \\
\hline & q15. locomoção & $\begin{array}{l}\text { Quão bem você é capaz de se locomover, isto é, caminhar com as próprias } \\
\text { pernas ou deslocar-se com a ajuda de aparelhos ou cadeira de rodas? }\end{array}$ & Muito mal a Muito bem \\
\hline & q16. sono & O quanto você está satisfeito(a) com o seu sono? & $\begin{array}{l}\text { Muito insatisfeito a } \\
\text { Muito satisfeito }\end{array}$ \\
\hline & q17. atividades diárias & $\begin{array}{l}\text { O quanto você está satisfeito(a) com sua capacidade de desempenhar as } \\
\text { atividades do seu dia a dia? }\end{array}$ & $\begin{array}{l}\text { Muito insatisfeito a } \\
\text { Muito satisfeito }\end{array}$ \\
\hline & $\begin{array}{l}\text { q18. capacidade } \\
\text { trabalho }\end{array}$ & O quanto você está satisfeito(a) com sua capacidade para o trabalho? & $\begin{array}{l}\text { Muito insatisfeito a } \\
\text { Muito satisfeito }\end{array}$ \\
\hline \multirow[t]{6}{*}{ Psicológico } & q5. aproveita vida & O quanto você aproveita a vida? & Nada a Extremamente \\
\hline & q6. sentido da vida & Em que medida você acha que a sua vida tem sentido? & Nada a Extremamente \\
\hline & q7. concentração & O quanto você consegue se concentrar? & Nada a Extremamente \\
\hline & q11. aparência física & Você é capaz de aceitar sua aparência física? & Nada a Completamente \\
\hline & q19. autossatisfação & O quanto você está satisfeito(a) consigo mesmo? & $\begin{array}{l}\text { Muito insatisfeito a } \\
\text { Muito satisfeito }\end{array}$ \\
\hline & $\begin{array}{l}\text { q26. sentimentos } \\
\text { negativos }\end{array}$ & $\begin{array}{l}\text { Com que frequência você tem sentimentos negativos tais como mau humor, } \\
\text { desespero, ansiedade, depressão? }\end{array}$ & Nunca a Sempre \\
\hline \multirow[t]{3}{*}{$\begin{array}{l}\text { Relações } \\
\text { sociais }\end{array}$} & q20. relações pessoais & $\begin{array}{l}\text { Quão satisfeito você está com suas relações pessoais (amigos, parentes, } \\
\text { conhecidos, colegas)? }\end{array}$ & $\begin{array}{l}\text { Muito insatisfeito a } \\
\text { Muito satisfeito }\end{array}$ \\
\hline & q21. vida sexual & O quanto você está satisfeito(a) com sua vida sexual? & $\begin{array}{l}\text { Muito insatisfeito a } \\
\text { Muito satisfeito }\end{array}$ \\
\hline & q22. apoio dos amigos & O quanto você está satisfeito(a) com o apoio que você recebe de seus amigos? & $\begin{array}{l}\text { Muito insatisfeito a } \\
\text { Muito satisfeito }\end{array}$ \\
\hline Meio & q8. segurança na vida & O quanto você se sente seguro(a) em sua vida diária? & Nada a Extremamente \\
\hline \multirow[t]{7}{*}{ ambiente } & 99. ambiente saudável & O quanto o seu ambiente físico é saudável (clima, barulho, poluição, atrativos)? & Nada a Extremamente \\
\hline & $\begin{array}{l}\text { q12. recursos } \\
\text { financeiros }\end{array}$ & Você tem dinheiro suficiente para satisfazer suas necessidades? & Nada a Completamente \\
\hline & $\begin{array}{l}\text { 913. informaç̃̃es } \\
\text { disponiveis }\end{array}$ & $\begin{array}{l}\text { O quanto as informações que precisa no seu dia a dia estão disponíveis para } \\
\text { você? }\end{array}$ & Nada a Completamente \\
\hline & q14. atividades de lazer & Em que medida você tem oportunidades de atividade de lazer? & Nada a Completamente \\
\hline & q23. moradia & O quanto você está satisfeito(a) com as condições do local onde mora? & $\begin{array}{l}\text { Muito insatisfeito a } \\
\text { Muito satisfeito }\end{array}$ \\
\hline & $\begin{array}{l}\text { q24. acesso serviços de } \\
\text { saúde }\end{array}$ & O quanto você está satisfeito(a) com o seu acesso aos serviços de saúde? & $\begin{array}{l}\text { Muito insatisfeito a } \\
\text { Muito satisfeito }\end{array}$ \\
\hline & $\begin{array}{l}\text { q25. meio de } \\
\text { transporte }\end{array}$ & O quanto você está satisfeito(a) com o seu meio de transporte? & $\begin{array}{l}\text { Muito insatisfeito a } \\
\text { Muito satisfeito }\end{array}$ \\
\hline
\end{tabular}

* Disponivel em http://www.ufrgs.br/psiq/whoqol86.html (acessado em 30/Jun/2010);

** Os escores variam de 1 a 5, à exceção dos itens q3, q26 e q4 (pontuação inversa). 
Tabela 2

Descrição dos achados dos estudos de validade dimensional do WHOQOL-BREF.

\begin{tabular}{|c|c|c|c|c|c|c|c|c|c|}
\hline \multirow[t]{2}{*}{ Autor, ano (país) } & \multirow[t]{2}{*}{ População (n) } & \multirow{2}{*}{$\begin{array}{l}\text { Número } \\
\text { dos itens }\end{array}$} & \multirow[t]{2}{*}{ Técnica } & \multirow{2}{*}{$\begin{array}{l}\text { Número } \\
\text { de fatores }\end{array}$} & \multicolumn{4}{|c|}{ Indicadores de ajuste } & \multirow{2}{*}{$\begin{array}{l}\text { Modelo de } \\
\text { correlações }\end{array}$} \\
\hline & & & & & RMSEA & CFI & TLI & Outros & \\
\hline $\begin{array}{l}\text { Chachamovich et al. 21, } 2009 \\
\text { (Brasil) }\end{array}$ & Idosos (1.048) & 24 & AFC & 4 & 0,07 & 0,88 & 0,86 & - & 1a ordem \\
\hline Chen et al. 32, 2006 (China) & Adolescentes (365) & 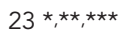 & AFC & 4 & 0,07 & 0,96 & - & - & 2a ordem \\
\hline \multirow[t]{2}{*}{ Chung et al. 22, 2012 (China) } & $\begin{array}{c}\text { Doentes de } \\
\text { tuberculose (140) }\end{array}$ & $23 *, \star \star, \#$ & AFC & 6 \#\#\# & 0,07 & 0,86 & - & $A I C=538,8$ & 1a ordem \\
\hline & Sadios (130) & $23 *, \star \star, \# \#$ & AFC & 4 \#\#\# & 0,08 & 0,86 & - & $A I C=501,6$ & 1a ordem \\
\hline Fang et al. 28, 2002 (China) & Doentes HIV (136) & 26 * & AFE (ACP, promax) & 4 & - & - & - & $47 \%$ VTE & 1a ordem \\
\hline Jaracz et al. 23, 2006 (Polônia) & Geral (908) & 24 & AFC & 4 \#\#\# & 0,06 & 0,87 & - & - & 1ạ ordem \\
\hline $\begin{array}{l}\text { Kalfoss et al. 18, } 2008 \\
\text { (Canadá) }\end{array}$ & Idosos (192) & 24 & AFC & 4 & 0,08 & 0,88 & - & - & 2a ordem \\
\hline (Noruega) & Idosos (469) & 24 & AFC & 4 \#\#\# & 0,08 & 0,81 & - & - & \\
\hline Li et al. 24, 2009 (Tailândia) & $\begin{array}{l}\text { Estudantes de } \\
\text { graduação (407) }\end{array}$ & 24 & $\mathrm{AFC}$ & 4 & 0,05 & 0,97 & - & - & $\begin{array}{l}\text { 2a ordem } \\
q^{3} \Leftrightarrow q^{4} \\
q^{8} \Leftrightarrow q^{9}\end{array}$ \\
\hline Min et al. 17, 2002 (Corea) & $\begin{array}{l}\text { Sadios (367), } \\
\text { doentes (171) }\end{array}$ & 24 & AFC & 4 & - & 0,86 & - & - & 1a ordem \\
\hline Moreno et al. 20, 2006 (Brasil) & $\begin{array}{l}\text { Trabalhadores da } \\
\text { universidade (3.574) }\end{array}$ & 24 & $\mathrm{AFE}(\mathrm{ACP}$, varimax $)$ & 4 \#\#\#,§ & - & - & - & $76 \%$ VTE & 1a ordem \\
\hline $\begin{array}{l}\text { Ohaeri \& Awadalla 5, } 2009 \\
\text { (Kuwait) }\end{array}$ & Geral (3.303) & 26 & $\begin{array}{l}\mathrm{AFE}(\mathrm{ACP}, \\
\text { ortogonal) }\end{array}$ & 5 & - & - & - & $59 \%$ VTE & 1a ordem \\
\hline $\begin{array}{l}\text { Ohaeri et al. 6, } 2007 \\
\text { (Arábia Saudita) }\end{array}$ & Geral (623) & 26 & AFC & 6 & 0,06 & - & - & $A G F I=0,86$ & 1a ordem \\
\hline $\begin{array}{l}\text { von Steinbüchel et al. 26, } \\
2006 \text { (Suiça) }\end{array}$ & Idosos (262) & 24 & AFC & 4 & 0,09 & 0,92 & - & - & 1ạ ordem \\
\hline $\begin{array}{l}\text { Taylor et al. 29, } 2004 \\
\text { (Austrália) }\end{array}$ & Doentes (72) & 24 & $\mathrm{AFE}(\mathrm{ACP}$, varimax) & 4 & - & - & - & $53 \%$ VTE & 1a ordem \\
\hline $\begin{array}{l}\text { Trompenaars et al. 30, } 2005 \\
\text { (Holanda) }\end{array}$ & $\begin{array}{c}\text { Doentes } \\
\text { psiquiátricos (495) }\end{array}$ & 25 ** & $\mathrm{AFE}(\mathrm{ACP}$, varimax) & 4 & - & - & - & - & 1a ordem \\
\hline $\begin{array}{l}\text { Skevington et al. 25, } 2004 \\
\text { (multicêntrico) }\end{array}$ & Geral (11.830) & 24 & AFC & 4 & 0,07 & 0,86 & - & - & 2a ordem \\
\hline Usefy et al. 31, 2010 (Irã) & Adultos (5.892) & 24 & $\mathrm{AFE}(\mathrm{ACP}$, varimax) & 4 & - & - & - & $50 \%$ VTE & 1ạ ordem \\
\hline $\begin{array}{l}\text { World Health } \\
\text { Organization 19, } 1998 \\
\text { (multicêntrico) }\end{array}$ & Geral (2.369) & 24 & AFC & 4 & - & 0,90 & - & - & $\begin{array}{l}\text { 2a ordem } \\
q^{3} \Leftrightarrow q^{4} \\
q^{3} \Leftrightarrow q^{26} \\
q^{23} \Leftrightarrow q^{9}\end{array}$ \\
\hline Xia et al. 27, 2012 (China) & Geral (1.052) & 24 & AFC & 4 & 0,09 & 0,91 & - & $N N F I=0,90$ & $\begin{array}{l}\text { 1ạ ordem } \\
q^{5} \Leftrightarrow q^{6} \\
q^{8} \Leftrightarrow q^{9}\end{array}$ \\
\hline
\end{tabular}

ACP: análise de componentes principais; AFC: análise fatorial confirmatória; AFE: análise fatorial exploratória; AGFI: Adjusted Goodness of Fit Index; AIC: Akaike's Information Criterion; CFI: Comparative Fit Index; NNFI: Non-Normed Fit Index; RMSEA: Root Mean Square Error of Approximation; TLI: Tucker-Lewis index; VTE: variância total explicada; q3: "dor física"; q4: "tratamento"; q5: "aproveita a vida"; q6: "sentido da vida"; q8: "segurança na vida"; q9: "ambiente saudável".

*Acrescentou dois itens regionais ao WHOQOL-BREF;

** Retirou dois itens da faceta geral;

*** Excluiu três itens (q3, q4 e q21 "vida sexual");

\# Excluiu três itens (q3, q4 e q5);

\#\# Excluiu quatro itens (q3, q4, q16 "sono" e q22 "apoio dos amigos";

\#\#\# Sem correspondência com o WHOQOL-BREF;

$\S$ Itens com cargas em mais de um fator. 
e q26 "sentimentos negativos" com pontuação inversa (Tabela 1).

O WHOQOL-BREF foi autopreenchido sob supervisão de equipe treinada em sala reservada. O estudo foi aprovado no comitê de ética em pesquisa sob o número CAE 0024.0.009.000-03.

\section{Análise de dados}

A descrição da população do estudo por meio de variáveis sociodemográficas foi realizada no programa estatístico SPSS, versão 17 (SPSS Inc., Chicago, Estados Unidos).

A análise foi subdividida em quatro etapas. Na primeira, foram testados dois modelos, via análise fatorial confirmatória, um com 24 itens e outro com 26 itens, incluindo os dois itens gerais. Se os índices de ajuste fossem inadequados, previu-se, em uma segunda etapa, realizar análise fatorial exploratória com todos os 26 itens para identificar modelos alternativos com significado teórico. A terceira etapa consistiu de nova análise fatorial confirmatória aplicada ao melhor modelo com 26 itens sugerido pela análise fatorial exploratória. Por fim, avaliou-se a validade fatorial convergente e discriminante por meio da variância média extraída e correlação entre fatores.

As análises fatoriais foram realizadas no software Mplus, versão 5.2 (Muthén \& Muthén, Los Angeles, Estados Unidos). A análise fatorial confirmatória tem como princípio a confirmação da estrutura fatorial proposta teoricamente. A estimação dos parâmetros do modelo foi realizada por meio do método dos mínimos quadrados ponderados robustos WLSMV, que utiliza matrizes de correlação policórica, adequadas para itens com opções de resposta categóricas ou ordinais 34 , como os itens do WHOQOL-BREF (Tabela 1). Cargas fatoriais $(\lambda)>0,40$ foram consideradas satisfatórias. Os erros de mensuração ( $\delta) \geq 0$,70 foram considerados altos e inadequa dos 35 . A qualidade do ajuste do modelo foi avaliada por meio de três índices: os índices de ajuste incremental CFI $>0,90$ e Tuckey-Lewis index (TLI) $>0,90$ e o índice de ajuste parcimonioso RMSEA < 0,08 34. Estes índices sugerem adequação do ajuste geral do modelo, mas não prescindem de uma avaliação do significado teórico ou mesmo da avaliação da validade preditiva do modelo final 35 . Os intervalos de $95 \%$ de confiança (IC95\%) foram estimados via bootstrap.

Para a re-especificação de todos os modelos, foram inspecionados os índices de modificação $(\mathrm{IM}>0,10)$ que refletem o quanto o $\chi^{2}$ do modelo poderia ser reduzido se um parâmetro fosse livremente estimado para avaliar itens com cargas potenciais em domínios não teoricamente con- siderados ou correlações entre resíduos de pares de itens 35 .

Adicionalmente, foi prevista uma segunda etapa consistindo de análise fatorial exploratória aplicada a todos os 26 itens do WHOQOL-BREF, utilizando o método de fatores comuns com rotação oblíqua geomin e o estimador WLSMV (adequado a dados ordinais), para identificar modelos alternativos a serem testados por meio de análise fatorial confirmatória 36 . Os índices de ajuste estimados foram CFI, TLI, RMSEA e SRMR (standardized root mean residual). Brown 35 considera que a análise fatorial exploratória serve de apoio para uma subsequente análise fatorial confirmatória ao investigar o comportamento dos itens e a estrutura multidimensional proposta 37 . Assim, a terceira etapa consistiu em nova análise fatorial confirmatória aplicada ao modelo de 26 itens sugeridos na etapa anterior (análise fatorial exploratória).

A consistência interna foi avaliada pela confiabilidade composta, visto que o alfa de Cronbach subestima a confiabilidade em situações de violação da tal equivalência, em que todos os itens deveriam ter as mesmas cargas fatoriais mesmo que suas variâncias únicas sejam diferentes. A confiabilidade composta varia de 0 a 1 sendo considerado satisfatório quando confiabilidade composta $\geq 0,7035$

Na quarta etapa, foram avaliadas as validades fatorial convergente e discriminante, respectivamente, por: (1) magnitude das cargas fatoriais e avaliação da variância média extraída (VME) - que representa o percentual médio de variação explicada pelos itens do construto. Varia de 0 a 1 , sendo que valores de $\mathrm{VME} \geq 0,50$ sugerem que os itens compartilham de uma elevada variância em comum; (2) magnitude das correlações entre fatores $(\leq 0,80)$ e pela comparação da raiz quadrada da VME $(\sqrt{\mathrm{VME}})$ de determinado fator com a magnitude da correlação entre este fator e os demais do modelo 35 . A validade discriminante foi corroborada se a raiz quadrada da VME de um fator fosse maior do que as correlações entre este e os demais fatores 34 .

\section{Resultados}

Do total de 422 trabalhadores entrevistados, 386 responderam de modo completo ao WHOQOLBREF: $67,8 \%$ do sexo feminino, 49,5\% em união estável, média de idade de 36,7 anos (DP = 11; 19-67), 58,5\% de cor branca e 31,3\% com pósgraduação. Quanto à ocupação, 40,6\% eram dirigentes ou profissionais (médicos, enfermeiros, biólogos, psicólogos), 53,8\% eram técnicos de nível médio (técnicos de enfermagem, adminis- 
trativos) e 5,5\% trabalhadores não especializados (limpeza, manutenção). Aqueles com dados faltantes e não incluídos eram predominantemente do sexo feminino $(79,4 \%)$, tinham união estável $(41,9 \%)$, apresentavam média de idade $43,2$ anos (DP $=11,4 ; 23-62)$, cor branca $(25 \%)$, 2 o grau completo $(38,7 \%)$ e $39,4 \%$ trabalhadores não especializados.

Os resultados da análise fatorial confirmatória estão apresentados na Tabela 3. No primeiro modelo, com 24 itens distribuídos em quatro fatores, a maioria dos itens teve carga fatorial satisfatória $(\lambda>0,40)$, à exceção do item "locomoção" do domínio físico $\left(\lambda_{\mathrm{q} 15}=0,27\right)$. Entretanto, os erros de mensuração foram altos $(\delta \geq 0,70)$ para três de sete itens do físico ("dor física", "tratamento" e "locomoção"), dois de seis itens do psicológico ("sentido da vida" e "sentimentos negativos") e cinco de oito itens de meio ambiente ("ambiente saudável", "recursos financeiros", "informações disponíveis", "acesso a serviços de saúde" e "meio de transporte"). Os índices de ajuste do modelo não foram satisfatórios, à exceção do TLI $(>0,90)$. A correlação entre fatores variou de 0,54 (IC95\%: $0,45-0,62$ ) para físico $\Leftrightarrow$ relações sociais a 0,81 (IC95\%: 0,76-0,86) para físico $\Leftrightarrow$ psicológico (Tabela 4). Os IM mostraram que se estimássemos os itens q8 "segurança na vida" (meio ambiente) e q10 "energia" (físico) no domínio psicológico e incluíssemos as correlações entre os erros de mensuração dos itens q3 "dor física" com q4 "tratamento" e q17 "desempenho das atividades diárias" com q18 "capacidade para o trabalho", os valores do $\chi^{2}$ para este novo modelo decairiam com uma estimativa de mudança no valor da carga acima de 0,60. Ainda que a inclusão destes parâmetros no modelo corrobore as cargas dos itens q8 e q10 apenas no domínio psicológico $\left(\lambda_{\mathrm{q} 8}=0,66\right.$ e $\left.\lambda_{\mathrm{q} 10}=0,70\right)$ e as correlações entre resíduos ( $\mathrm{q} 3 \Leftrightarrow \mathrm{q} 4=0,58$ e $\mathrm{q} 17 \Leftrightarrow \mathrm{q} 18=0,52)$, o ajuste permaneceu insatisfatório $(\mathrm{RMSEA}=0,08, \mathrm{CFI}=$ 0,91 e TLI $=0,95$ ).

No segundo modelo de análise fatorial confirmatória com os 26 itens do WHOQOL-BREF distribuídos em cinco fatores (geral, físico, psicológico, relações sociais e meio ambiente), as cargas dos itens q1 "qualidade de vida" e q2 "saúde" mostraram-se adequadas para o fator geral. Assim como no modelo anterior, o item q15 "locomoção" teve carga fatorial baixa $\left(\lambda_{\mathrm{q} 15}=0,28\right)$. Os índices de ajuste não diferiram do modelo anterior (modelo 1, Tabela 3) e, similarmente, os índices de modificação sugeriram que os itens q8 "segurança na vida" e q10 "energia" tivessem cargas no domínio psicológico e uma correlação entre os erros de mensuração dos itens q3 "tratamento" e q4 "dor". Entretanto, a inclusão destes parâmetros não propiciou uma adequação dos índices de ajuste (RMSEA $=0,09, \mathrm{CFI}=0,88 \mathrm{e}$ $\mathrm{TLI}=0,95$ ), ainda que as cargas de q8 e q10 somente no domínio psicológico $\left(\lambda_{\mathrm{q} 8}=0,70\right.$ e $\lambda_{\mathrm{q} 10}=$ $0,54)$ e a correlação entre resíduos ( $\mathrm{q} 3 \Leftrightarrow \mathrm{q} 4: 0,56$ ) tenham se confirmado.

Foi, então, realizada análise fatorial exploratória de 26 itens cujos resultados não são apresentados em tabela. Nesta análise a solução mais adequada mostrou que apenas as dimensões relações sociais e geral corresponderam à proposta teórica do grupo WHOQOL. O domínio físico se subdividiu em dois fatores: físico 1, representado pelos itens "dor física", "tratamento" e "locomoção" e; físico 2 - "sono", "atividades diárias", "capacidade trabalho", além de um item proposto teoricamente pelo grupo WHOQOL para o domínio psicológico ("autossatisfação"). Os itens "aproveita vida" e "autossatisfação" tiveram cargas significativas não apenas no domínio psicológico, mas também nos domínios relações sociais $\left(\lambda_{\mathrm{q} 5}=0,31\right)$ e físico $2\left(\lambda_{\mathrm{q} 19}=0,44\right)$, respectivamente. "Segurança" (meio ambiente), "ambiente saudável" (meio ambiente) e "energia” (físico) apresentaram cargas fatoriais significativas no domínio psicológico, e não no domínio meio ambiente como proposto pela sintaxe original do grupo WHOQOL. Os itens da faceta geral "qualidade de vida" e "saúde" carregaram nos fatores correspondentes a meio ambiente e físico 1, respectivamente. Este modelo resultante desta análise fatorial exploratória mostrou índices de ajuste satisfatórios $(\mathrm{CFI}=0,93$, TLI $=$ 0,96, RMSEA $=0,07$ e SRMR $=0,04)$ e correlações fatoriais que oscilaram entre 0,26 (físico $1 \Leftrightarrow$ meio ambiente) e 0,51 (psicológico $\Leftrightarrow$ físico 2).

Baseado nos resultados desta análise fatorial exploratória com todos os 26 itens, um novo modelo com seis fatores foi proposto para ser avaliado na análise fatorial confirmatória: geral, físico 1, físico 2, psicológico, relações sociais e meio ambiente, em que os itens "energia" (físico) e "segurança na vida" (meio ambiente) foram remanejados para o domínio psicológico. Por questões teóricas, optamos por estimar as cargas de "qualidade de vida" e "saúde" em geral - preservando a ideia do grupo WHOQOL (Tabela1), "aproveitar a vida" e "autossatisfação" em psicológico e manter o item "ambiente saudável" em meio ambiente. Neste novo modelo, a carga estimada do item "ambiente saudável" melhorou (0,51; IC95\%: 0,41-0,61). A correlação entre os fatores oscilou de 0,24 (físico $1 \Leftrightarrow$ relações sociais; IC95\%: 0,11-0,38) a 0,75 (físico $2 \Leftrightarrow$ psicológico; IC95\%: 0,70-0,81) (dados não mostrados). Os índices de modificação mostraram que se o item "qualidade de vida" fosse estimado livremente em meio ambiente e "moradia" em psicológico e relações sociais e se as correlações entre os erros 
Tabela 3

Análise fatorial confirmatória do WHOQOL-BREF: cargas fatoriais $(\lambda)$, uniqueness ( $(\delta)$ e índices de ajuste.

\begin{tabular}{|c|c|c|c|c|c|c|c|c|c|}
\hline \multirow[t]{2}{*}{ Itens/Fatores } & \multicolumn{3}{|c|}{ Modelo 1 (24 itens) } & \multicolumn{3}{|c|}{ Modelo 2 (26 itens) } & \multicolumn{3}{|c|}{ Modelo 3 (26 itens) } \\
\hline & $\lambda$ & IC95\% & $\delta$ & $\lambda$ & IC95\% & $\delta$ & $\lambda$ & IC95\% & $\delta$ \\
\hline & & & & \multicolumn{3}{|c|}{ Geral } & \multicolumn{3}{|c|}{ Geral } \\
\hline q1. qualidade de vida & - & - & - & 0,72 & $0,63-0,80$ & 0,49 & 0,71 & $0,63-0,79$ & 0,50 \\
\hline \multirow[t]{2}{*}{ q2. saúde } & - & - & - & 0,62 & $0,54-0,71$ & 0,61 & 0,63 & $0,54-0,72$ & 0,60 \\
\hline & \multicolumn{3}{|c|}{ Físico } & \multicolumn{3}{|c|}{ Físico } & \multicolumn{3}{|c|}{ Físico 1} \\
\hline q3. dor física & 0,44 & $0,35-0,53$ & 0,80 & 0,47 & $0,38-0,56$ & 0,78 & 0,81 & $0,70-0,92$ & 0,34 \\
\hline q4. tratamento & 0,41 & $0,31-0,51$ & 0,83 & 0,44 & $0,34-0,54$ & 0,80 & 0,75 & $0,65-0,86$ & 0,44 \\
\hline q10. energia & 0,71 & $0,66-0,76$ & 0,50 & 0,71 & $0,66-0,76$ & 0,50 & - & - & - \\
\hline \multirow[t]{2}{*}{ q15. locomoção } & 0,27 & $0,13-0,41$ & 0,93 & 0,28 & $0,15-0,42$ & 0,92 & 0,45 & $0,28-0,62$ & 0,80 \\
\hline & & & & & & & \multicolumn{3}{|c|}{ Físico 2} \\
\hline q16. sono & 0,60 & $0,53-0,68$ & 0,63 & 0,61 & $0,54-0,68$ & 0,63 & 0,64 & $0,56-0,71$ & 0,60 \\
\hline q17. atividades diárias & 0,88 & $0,84-0,92$ & 0,24 & 0,88 & $0,84-0,91$ & 0,23 & 0,90 & $0,86-0,94$ & 0,19 \\
\hline \multirow[t]{2}{*}{ q18. capacidade trabalho } & 0,89 & $0,85-0,92$ & 0,21 & 0,89 & $0,85-0,92$ & 0,22 & 0,91 & $0,87-0,95$ & 0,17 \\
\hline & \multicolumn{3}{|c|}{ Psicológico } & \multicolumn{3}{|c|}{ Psicológico } & \multicolumn{3}{|c|}{ Psicológico } \\
\hline q5. aproveita vida & 0,56 & $0,49-0,63$ & 0,68 & 0,58 & $0,50-0,65$ & 0,67 & 0,55 & $0,48-0,62$ & 0,70 \\
\hline q6. sentido da vida & 0,52 & $0,44-0,60$ & 0,73 & 0,52 & $0,44-0,60$ & 0,73 & 0,53 & $0,45-0,61$ & 0,72 \\
\hline q7. concentração & 0,62 & $0,54-0,69$ & 0,62 & 0,61 & $0,53-0,68$ & 0,63 & 0,57 & $0,49-0,65$ & 0,68 \\
\hline q11. aparência física & 0,62 & $0,55-0,68$ & 0,62 & 0,62 & $0,55-0,69$ & 0,62 & 0,63 & $0,56-0,70$ & 0,61 \\
\hline q19. autossatisfação & 0,80 & $0,75-0,85$ & 0,36 & 0,80 & $0,74-0,85$ & 0,37 & 0,81 & $0,76-0,86$ & 0,34 \\
\hline q26. sentimentos negativos & 0,51 & $0,42-0,59$ & 0,74 & 0,51 & $0,42-0,59$ & 0,74 & 0,52 & $0,44-0,61$ & 0,73 \\
\hline q8. segurança na vida * & - & - & - & - & - & - & 0,62 & $0,55-0,70$ & 0,61 \\
\hline \multirow[t]{2}{*}{ q10. energia * } & - & - & - & - & - & - & 0,70 & $0,65-0,76$ & 0,51 \\
\hline & \multicolumn{3}{|c|}{ Relações sociais } & \multicolumn{3}{|c|}{ Relações sociais } & \multicolumn{3}{|c|}{ Relações sociais } \\
\hline q20. relações pessoais & 0,93 & $0,88-0,99$ & 0,13 & 0,93 & $0,88-0,99$ & 0,13 & 0,94 & $0,89-0,99$ & 0,11 \\
\hline q21. vida sexual & 0,62 & $0,53-0,70$ & 0,62 & 0,62 & $0,54-0,71$ & 0,62 & 0,60 & $0,51-0,69$ & 0,64 \\
\hline \multirow[t]{2}{*}{ q22. apoio dos amigos } & 0,68 & $0,61-0,74$ & 0,54 & 0,67 & $0,61-0,74$ & 0,55 & 0,67 & $0,61-0,74$ & 0,54 \\
\hline & \multicolumn{3}{|c|}{ Meio ambiente } & \multicolumn{3}{|c|}{ Meio ambiente } & & leio ambien & \\
\hline q8. segurança na vida & 0,71 & $0,63-0,79$ & 0,49 & 0,71 & $0,63-0,79$ & 0,50 & - & - & - \\
\hline q9. ambiente saudável & 0,48 & $0,38-0,58$ & 0,77 & 0,48 & $0,38-0,57$ & 0,77 & 0,51 & $0,41-0,61$ & 0,74 \\
\hline q12. recursos financeiros & 0,52 & $0,44-0,60$ & 0,73 & 0,53 & $0,45-0,61$ & 0,72 & 0,59 & $0,51-0,68$ & 0,65 \\
\hline q13. informações disponíveis & 0,49 & $0,40-0,57$ & 0,76 & 0,48 & $0,40-0,57$ & 0,77 & 0,53 & $0,45-0,62$ & 0,71 \\
\hline q14. atividades de lazer & 0,58 & $0,51-0,66$ & 0,66 & 0,59 & $0,52-0,66$ & 0,65 & 0,64 & $0,54-0,70$ & 0,62 \\
\hline q23. moradia & 0,63 & $0,56-0,70$ & 0,60 & 0,62 & $0,56-0,70$ & 0,61 & 0,68 & $0,60-0,75$ & 0,54 \\
\hline q24. acesso a serviços de saúde & 0,50 & $0,41-0,59$ & 0,75 & 0,50 & $0,42-0,59$ & 0,74 & 0,55 & $0,47-0,64$ & 0,70 \\
\hline q25. meio de transporte & 0,48 & $0,40-0,57$ & 0,74 & 0,48 & $0,39-0,56$ & 0,77 & 0,52 & $0,44-0,61$ & 0,73 \\
\hline Correlações entre itens & & & & & & & & & \\
\hline$q 5 \Leftrightarrow q 14$ & - & - & - & - & - & - & & 0,36 & - \\
\hline$q 5 \Leftrightarrow q 21$ & - & - & - & - & - & - & & 0,30 & - \\
\hline$q 7 \Leftrightarrow q 8$ & - & - & - & - & - & - & & 0,36 & - \\
\hline Índices de ajuste & & & & & & & & & \\
\hline RMSEA & & 0,11 & & & 0,11 & & & 0,07 & \\
\hline $\mathrm{CFI} / \mathrm{TLI}$ & & $0,83 / 0,91$ & & & $0,81 / 0,91$ & & & $0,92 / 0,96$ & \\
\hline
\end{tabular}

CFI: comparative fit index; RMSEA: Root Mean Square Error of Approximation; TLI: Tucker-Lewis index.

Modelo 1: físico $\Leftrightarrow$ psicológico $\Leftrightarrow$ relações sociais $\Leftrightarrow$ meio ambiente; modelo 2: geral $\Leftrightarrow$ físico $\Leftrightarrow$ psicológico $\Leftrightarrow$ relações sociais $\Leftrightarrow$ meio ambiente;

modelo 3: geral $\Leftrightarrow$ físico $1 \Leftrightarrow$ físico $2 \Leftrightarrow$ psicológico $(+q 8$ e q10) $\Leftrightarrow$ relações sociais $\Leftrightarrow$ meio ambiente com correlações entre q5 $\Leftrightarrow q 14, q 5 \Leftrightarrow q 21$ e q7 $\Leftrightarrow q 8$.

* Itens alocados em novos fatores baseado na análise fatorial exploratória. 
Análise fatorial confirmatória do WHOQOL-BREF: correlação entre os fatores.

\begin{tabular}{|c|c|c|c|}
\hline Correlações entre fatores & Modelo 1 (24 itens) & Modelo 2 (26 itens) & Modelo3 (26 itens) \\
\hline Geral $\Leftrightarrow$ Físico & - & 0,73 & - \\
\hline Geral $\Leftrightarrow$ Psicológico & - & 0,72 & 0,74 \\
\hline Geral $\Leftrightarrow$ Social & - & 0,54 & 0,54 \\
\hline Geral $\Leftrightarrow$ Ambiente & - & 0,74 & 0,70 \\
\hline Físico $\Leftrightarrow$ Psicológico & 0,81 & 0,80 & - \\
\hline Físico $\Leftrightarrow$ Ambiente & 0,63 & 0,63 & - \\
\hline Físico $\Leftrightarrow$ Social & 0,54 & 0,53 & - \\
\hline Psicológico $\Leftrightarrow$ Ambiente & 0,80 & 0,80 & 0,63 \\
\hline Psicológico $\Leftrightarrow$ Social & 0,75 & 0,75 & 0,71 \\
\hline Social $\Leftrightarrow$ Ambiente & 0,58 & 0,58 & 0,53 \\
\hline Físico $1 \Leftrightarrow$ Geral & - & - & 0,66 \\
\hline Físico $1 \Leftrightarrow$ Físico 2 & - & - & 0,40 \\
\hline Físico $1 \Leftrightarrow$ Psicológico & - & - & 0,36 \\
\hline Físico $1 \Leftrightarrow$ Ambiente & - & - & 0,36 \\
\hline Físico $1 \Leftrightarrow$ Social & - & - & 0,24 \\
\hline Físico $2 \Leftrightarrow$ Geral & - & - & 0,63 \\
\hline Físico $2 \Leftrightarrow$ Psicológico & - & - & 0,77 \\
\hline Físico $2 \Leftrightarrow$ Ambiente & - & - & 0,52 \\
\hline Físico $2 \Leftrightarrow$ Social & - & - & 0,51 \\
\hline
\end{tabular}

Modelo 1: físico $\Leftrightarrow$ psicológico $\Leftrightarrow$ relações sociais $\Leftrightarrow$ meio ambiente; modelo 2: geral $\Leftrightarrow$ físico $\Leftrightarrow$ psicológico $\Leftrightarrow$ relações sociais $\Leftrightarrow$ meio ambiente; modelo 3: geral $\Leftrightarrow$ físico $1 \Leftrightarrow$ físico $2 \Leftrightarrow$ psicológico (+ q8 "segurança" e q10 "energia") $\Leftrightarrow$ relações sociais $\Leftrightarrow$ meio ambiente, com correlações entre $q 5 \Leftrightarrow q 14, q 5 \Leftrightarrow q 21$ e q7 $\Leftrightarrow q 8$.

de mensuração de q7 "segurança” e q8 "concentração", q5 "aproveitar a vida" e q14 "atividades de lazer" e de q5 "aproveitar a vida" e q21 "vida sexual" fossem permitidas, o $\chi^{2}$ do modelo diminuiria. Por questões teóricas, conforme informado anteriormente, mantivemos o item q1 "qualidade de vida" somente na faceta geral. Quando avaliamos as cargas cruzadas sugeridas para o item "moradia” em psicológico e relações sociais, as mesmas não se confirmaram $(\leq 0,31)$. Entretanto, houve confirmação das correlações entre os resíduos de q7 e q8, q5 e q14 e de q5 e q2 adicionadas ao modelo final $(0,36,0,36$ e 0,30 , respectivamente), com melhora dos seus índices de ajuste: RMSEA $=0,07$, CFI $=0,92$ e TLI $=0,96$ (Tabela 3, modelo 3).

A consistência interna foi boa, à exceção do fator geral $(C C<0,70)$ nos modelos 2 e 3 . Entretanto, a validade fatorial convergente e discriminante só foi adequada para o domínio relações sociais, em todos os modelos, e físico 1 e físico 2 , no modelo 3 (Tabela 5).

\section{Discussão}

A estrutura fatorial de melhor ajuste para os 26 itens mostrou seis fatores (geral, físico 1, físico 2, psicológico, relações sociais e meio ambiente), entre os quais apenas dois corresponderam exatamente ao que foi proposto teoricamente para a faceta geral e para o domínio relações sociais e outros dois fatores resultaram da subdivisão do domínio físico. Neste modelo final, o domínio físico se subdividiu em dois fatores e os itens "energia" e "segurança", propostos respectivamente para os domínios físico e meio ambiente, foram remanejados para o domínio psicológico. Foi obtido um modelo final apresentando cargas fatoriais adequadas para a maioria dos itens e quatro índices de ajuste satisfatórios quando comparado ao modelo WHOQOL-BREF com 24 itens, ainda que oito dos 26 itens tenham permanecido com erros de mensuração de magnitude elevada.

Este estudo possui como ponto forte ser o primeiro a avaliar a estrutura dimensional do WHOQOL-BREF em trabalhadores brasileiros de instituição de pesquisa em saúde que utilizou a 
Análise fatorial confirmatória do WHOQOL-BREF: confiabillidade composta (CC) e variância média extraída (VME).

\begin{tabular}{lccccccccc}
\hline Fatores & \multicolumn{3}{c}{ Modelo 1 (24 itens) } & \multicolumn{3}{c}{ Modelo 2 (26 itens) } & \multicolumn{3}{c}{ Modelo3 (26 itens) } \\
& CC & VME & $\sqrt{\mathrm{VME}}$ & $\mathrm{CC}$ & $\mathrm{VME}$ & $\sqrt{\mathrm{VME}}$ & $\mathrm{CC}$ & VME & $\sqrt{\mathrm{VME}}$ \\
\hline Geral & - & - & - & 0,62 & 0,45 & 0,67 & 0,62 & 0,45 & 0,67 \\
Físico & 0,81 & 0,41 & 0,64 & 0,81 & 0,41 & 0,64 & - & - & - \\
Físico 1 & - & - & - & - & - & - & 0,72 & 0,47 & 0,69 \\
Físico 2 & - & - & - & - & - & - & 0,86 & 0,68 & 0,82 \\
Psicológico & 0,81 & 0,37 & 0,61 & 0,81 & 0,37 & 0,61 & 0,80 & 0,34 & 0,58 \\
Social & 0,79 & 0,57 & 0,65 & 0,79 & 0,57 & 0,75 & 0,79 & 0,57 & 0,75 \\
Ambiente & 0,78 & 0,31 & 0,56 & 0,78 & 0,31 & 0,56 & 0,77 & 0,33 & 0,58 \\
\hline
\end{tabular}

Modelo 1: físico $\Leftrightarrow$ psicológico $\Leftrightarrow$ relações sociais $\Leftrightarrow$ meio ambiente; modelo 2: geral $\Leftrightarrow$ físico $\Leftrightarrow$ psicológico $\Leftrightarrow$ relações

sociais $\Leftrightarrow$ meio ambiente; modelo 3: geral $\Leftrightarrow$ físico $1 \Leftrightarrow$ f́́sico $2 \Leftrightarrow$ psicológico (+ q8 "segurança" e q10 "energia") $\Leftrightarrow$ relações

sociais $\Leftrightarrow$ meio ambiente, com correlações entre $q 5 \Leftrightarrow q 14, q 5 \Leftrightarrow q 21$ e q7 $\Leftrightarrow q 8$.

análise fatorial confirmatória, técnica estatística que permitiu reavaliar o ajuste dos dados ao modelo teórico proposto pela OMS 2, pela utilização de um método estatístico mais apropriado para dados ordinais e categóricos e que apresentou resultados relativos à inspeção dos índices de modificação 35 .

Também, é o primeiro que avalia a consistência interna do WHOQOL-BREF por meio da confiabilidade composta, ao contrário dos estudos encontrados na literatura que utilizaram o alfa de Cronbach 5,17,20,23,31. Da mesma forma, avaliou a validade fatorial convergente e discriminante baseada na inspeção da variância média extraída e correlação entre fatores. A falta de validade fatorial convergente e discriminante no modelo final sugere má especificação dos domínios psicológico, geral e meio ambiente. Isto significa que estes domínios não funcionam conforme a proposição teórica do grupo WHOQOL, limitando o uso destes escores em estudos de associação. Assim, a revisão da semântica, ou mesmo substituição, dos itens "segurança na vida", "ambiente saudável”, "aproveita a vida” e "autossatisfação" poderia melhorar a especificidade da carga fatorial nos domínios postulados e, consequentemente, a validade destes construtos teóricos.

A inclusão dos 26 itens do WHOQOL-BREF na análise permitiu avaliar o papel dos dois itens propostos para a faceta geral no construto qualidade de vida. Por fim, o tamanho amostral está de acordo com o recomendado para modelos com dados categóricos 35 e foi similar ou superior a outros estudos de validação deste instrumento $18,26,28,29,32$.

Nossos resultados mostram que dos seis fatores obtidos, apenas dois claramente correspon- dem ao que foi proposto teoricamente. A subdivisão dos itens para o domínio físico em dois fatores se assemelha à distribuição dos itens do WHOQOL-100 nos domínios físico, composto pelas facetas "dor e desconforto", "energia e fadiga", "sono e repouso", e nível de independência pelas facetas "locomoção", "atividades diárias", "tratamento" e "capacidade para o trabalho" 2,19. Em nossa amostra de trabalhadores, a distribuição dos itens nos fatores físico 1 ("dor", "tratamento" e "locomoção") e físico 2 ("capacidade trabalho", "atividades diárias" e "sono") foi semelhante à encontrada por Moreno et al. ${ }^{20}$ por meio da análise fatorial exploratória, em uma amostra de trabalhadores administrativos de uma universidade pública, renomeados de "físico patológico" e "físico funcional", respectivamente, sugerindo que este último esteja relacionado ao nível de independência em uma população economicamente ativa.

Em nosso estudo, o modelo análise fatorial confirmatória utilizando somente 24 itens, distribuídos nos quatro domínios propostos pelo grupo WHOQOL (físico, psicológico, relações sociais e meio ambiente), apresentou pior ajuste comparado com outros estudos 17,18,21,25,26. Entendendo que a exclusão da faceta geral ("qualidade de vida" e "saúde") poderia acarretar em perda de informação relevante 6 , optamos por incluíla na análise fatorial confirmatória, entretanto, os índices de ajuste mantiveram-se semelhantes comparados ao modelo AFC com 24 itens na distribuição que segue a sintaxe divulgada pelo WHOQOL.

Outros estudos também demonstram a possibilidade de estruturas alternativas pela análise fatorial. Em uma amostra geral da população 
árabe, o WHOQOL-BREF ajustou-se com cinco fatores (58,7\% da variância) e os itens "tratamento", "dor" e "sentimentos negativos" carregaram em um fator denominado de "necessidades de cuidados da saúde" 5 . Outro fator "saúde física geral e qualidade de vida total" reuniu os demais itens do domínio físico do WHOQOL-BREF e as duas questões gerais de saúde e qualidade de vida. De modo similar ao nosso estudo, os fatores psicológico, relação social e meio ambiente englobaram os mesmos itens do modelo teoricamente proposto para o WHOQOL-BREF 19,21,25. Em três subgrupos da população sudanesa (geral, pacientes psiquiátricos e cuidadores de família), a análise fatorial confirmatória do WHOQOLBREF comparou modelos propostos pelo grupo WHOQOL (4 e 6 domínios) com os obtidos na análise fatorial exploratória dos dados empíricos. Os melhores ajustes foram obtidos na amostra de pacientes psiquiátricos para os modelos WHOQOL de quatro fatores (24 itens, domínios: físico, psicológico, relações sociais e meio ambiente) e de cinco fatores obtidos na análise fatorial exploratória com 26 itens, dos quais dois corresponderam aos domínios relações sociais e meio ambiente do WHOQOL e três novos fatores foram denominados: "satisfação com a vida" incluindo dez itens, quatro propostos no domí nio físico, quatro no psicológico e dois no meio ambiente; "sensação de prazer" - com cinco itens, dos quais dois da faceta geral, um do físico ("sono"), um do psicológico ("aproveita a vida") e um do meio ambiente ("atividades de lazer"); e "saúde física e mental" - com três itens, dois do físico ("dor" e "tratamento") e um do psicológico ("sentimentos negativos") 6 , semelhante ao fator denominado "necessidade de cuidados de saúde" em amostra da população árabe 5 .

No presente trabalho, os itens "segurança" e "energia" apresentaram maior carga no fator psicológico pela provável ambiguidade de suas argumentações. A pergunta "Quão seguro você se sente em sua vida diária?" pode abarcar tanto a percepção de "ações" ou "recursos" disponíveis para proteger alguém, diminuindo os perigos ou os riscos de danos físicos (em determinadas atividades laborativas, por exemplo), senso de liberdade (pela ausência de restrições), como também sentimento de crença em si mesmo ou de força interior. Da mesma forma, a questão " $V o$ cê tem energia suficiente para o seu dia-a-dia?" abrange o sentido de resistência física/cansaço para as atividades da vida diária, mas também pode ser interpretada pela "sensação de sentirse vivo" 38. Outros estudos encontraram achados similares para os itens "energia" 23,28,29,31 e "segurança” 20,23,30. Os itens q5 “aproveitar a vida” e q19 "autossatisfação" apresentaram cargas tanto no seu fator psicológico como em relações sociais e físico 2, respectivamente. A opção em mantê-los no fator psicológico justifica-se pela própria teoria em que "aproveitar vida" avalia sentimentos positivos de contentamento e "autossatisfação" abrange a percepção do indivíduo sobre seu autocontrole e direção na vida, não incluindo aspectos físicos (imagem corporal) e sociais 38 .

Estudo de fatores associados aos escores do WHOQOL-BREF realizado em estudantes universitários sugeriu possível intercambialidade do significado dos itens e que os domínios possam não ser construtos diferentes, mas redundantes 7 .

As sugestões para reespecificação do nosso melhor modelo foram aceitáveis teórica e empiricamente, com melhora de seus índices de ajuste, conforme demonstraram outros estudos. A covariância entre os itens "dor" e "tratamento' encontrada em nosso estudo nos modelos do WHOQOL-BREF de 24 e 26 itens é consistente com outros achados 19,24, mas não foi corroborada no nosso melhor modelo (modelo 3). Em estudantes de faculdade na Tailândia, Li et al. 24 encontraram índices de ajuste adequados do WHOQOL-BREF após permitir a covariância de erros entre os itens "dor" e "tratamento"; "segurança" e "ambiente saudável". Em pesquisa multicêntrica do WHOQOL, o índice de ajuste CFI foi limítrofe $(0,87)$ para um modelo com quatro domínios, que aumentou para 0,90 após permitir-se a covariância de erros entre três pares de itens ("dor" e "tratamento"; "dor" e "sentimentos negativos", "local de moradia" e "ambiente saudável") e cargas cruzadas de dois itens em outros domínios para o qual não haviam sido propostos teoricamente: "segurança" e geral; "tratamento" e meio ambiente) 19

O modelo do WHOQOL-BREF considera que seus quatro domínios, hipotetizados como de primeira ordem, sejam influenciados por um construto geral hierárquico de qualidade de vida (segunda ordem), com explicação mais parcimoniosa das correlações entre os seus dominios. Entretanto, em nossa amostra, houve inviabilidade empírica para testarmos modelos de segunda ordem de fator único pelo mau ajuste do modelo de primeira ordem e pelas diferentes magnitudes das correlações entre os fatores, apesar dos seus altos valores 35 . De forma semelhante, dois estudos assumiram modelos do WHOQOL com ajustes aceitáveis caracterizados como de primeira ordem em população de idosos 21,26.

A principal limitação deste estudo refere-se ao escopo profissional da população alvo, restrita a uma instituição de saúde da cidade do Rio de Janeiro que, no entanto, incluiu representantes de seis dos nove grandes grupos da classificação 
brasileira de ocupações ${ }^{39}$. Assim, a generalização externa abrange o grupo de melhor escolaridade e nível ocupacional.

Estudos de dimensionalidade para este instrumento são relevantes considerando não só o crescente uso do mesmo no Brasil 7,8,9,20,33, desacompanhado de equivalente esforço na sedimentação de seus domínios para diferentes populações e as consequentes implicações para a sintaxe, cálculo de escores e ações que possam ser desencadeadas sem a devida reflexão sobre a intercambialidade de itens entre fatores.

\section{Resumen}

El objetivo fue reevaluar la estructura dimensional de la versión brasileña del WHOQOL-BREF, consistencia interna y validez factorial convergente y discriminante. Se realizó un estudio por secciones en 386 trabajadores de salud. El análisis factorial confirmatorio comprobó la estructura del WHOQOL-BREF (24 y 26 items) y la sugerida por el análisis factorial exploratorio. La consistencia interna fue contrastada vía confiabilidad compuesta; validez convergente y discriminante, por la variancia media extraída y la correlación entre factores. El modelo de mejor ajuste fue el sugerido por el análisis factorial exploratorio (26 items) con seis factores: cuatro propuestos teóricamente (general, psicológico, relaciones sociales y medio ambiente) y dos por la subdivisión del dominio físico. Los ítems "energía" y "seguridad" (respectivamente, procedentes del físico y medio ambiente) fueron reorientados hacia el psicológico. La confiabilidad compuesta fue buena $(>0,70)$, con excepción del factor general. La validez convergente y discriminante fueron adecuadas para las relaciones sociales $y$ físicas. Persisten controversias sobre la dimensionalidad del WHOQOL-BREF, particularmente sobre el dominio físico.

Estudios de Validación; Personal de Salud; Calidad de Vida; Ambiente de Trabajo
Em síntese, este estudo confirmou apenas parcialmente o modelo de mensuração proposto teoricamente para o WHOQOL-BREF, particularmente para os domínios psicológico, relações sociais e meio ambiente. A subdivisão do domínio físico em dois componentes, denominados por Moreno et al. 20 como funcional e patológico pode sugerir uma interpretação diferente desta dimensão física por trabalhadores saudáveis da área de saúde, o que precisa ser corroborado em outros estudos com amostras semelhantes e em análises de múltiplos grupos.

\section{Colaboradores}

M. M. L. D. Castro, Y. H. M. Hökerberg e S. R. L. Passos participaram da concepção, da análise e interpretação dos dados, da redação e da revisão do artigo.

\section{Agradecimentos}

M. M. L. D. Castro recebeu bolsa de estudos da Fundação Oswaldo Cruz (Fiocruz). S. R. L. Passos foi contemplada com bolsa JCNE, no E-26/101511/2010, da FAPERJ. 


\section{Referências}

1. Skevington SM. Advancing cross-cultural research on quality of life: observations drawn from the WHOQOL development. World Health Organization Quality of Life Assessment. Qual Life Res 2002; 11:135-44.

2. The World Health Organization Quality of Life as sessment (WHOQOL): position paper from the World Health Organization. Soc Sci Med 1995; 41:1403-9.

3. What quality of life? The WHOQOL Group. World Health Organization Quality of Life Assessment. World Health Forum 1996; 17:354-6.

4. The World Health Organization Quality of Life Assessment (WHOQOL): development and general psychometric properties. Soc Sci Med 1998; 46:1569-85.

5. Ohaeri JU, Awadalla AW. The reliability and validity of the short version of the WHO Quality of Life Instrument in an Arab general population. Ann Saudi Med 2009; 29:98-104.

6. Ohaeri JU, Awadalla AW, El-Abassi AH, Jacob A. Confirmatory factor analytical study of the WHOQOL-BREF: experience with Sudanese general population and psychiatric samples. BMC Med Res Methodol 2007; 7:37.

7. Blay SL, Marchesoni MS. Association among physical, psychiatric and socioeconomic conditions and WHOQOL-BREF scores. Cad Saúde Pública 2011; 27:677-86.

8. Calumbi RA, Amorim JA, Maciel CMC, Damázio Filho O, Teles AJF. Evaluation of the quality of life of anesthesiologists in the city of Recife. Rev Bras Anestesiol 2010; 60:42-51.

9. Paschoa S, Zanei SSV, Whitaker IY. Qualidade de vida dos trabalhadores de enfermagem de unidades de terapia intensiva. Acta Paul Enferm 2007; 20:305-10.

10. Berlim MT, Mattevi BS, Fleck MP. Depression and quality of life among depressed Brazilian outpatients. Psychiatr Serv 2003; 54:254.

11. Berlim MT, Pargendler J, Brenner J, Fleck MP. Significant improvement in the quality of life of Brazilian depressed outpatients 12 weeks following the start of antidepressants. Psychiatry Res 2007; 153:253-9.

12. Gazalle FK, Andreazza AC, Cereser KM, Hallal PC Santin A, Kapczinski F. Clinical impact of late diagnose of bipolar disorder. J Affect Disord 2005; 86:313-6.

13. Rabin EG, Heldt E, Hirakata VN, Fleck MP. Quality of life predictors in breast cancer women. Eur J Oncol Nurs 2008; 12:53-7.

14. Romao AP, Gorayeb R, Romão GS, Poli-Neto OB, Reis FJ, Rosa-e-Silva JC, et al. High levels of anxiety and depression have a negative effect on quality of life of women with chronic pelvic pain. Int J Clin Pract 2009; 63:707-11.

15. Chachamovich E, Fleck M, Laidlaw K, Power M. Impact of major depression and subsyndromal symptoms on quality of life and attitudes toward aging in an international sample of older adults. Gerontologist 2008; 48:593-602.
16. Paskulin L, Vianna L, Molzahn AE. Factors associated with quality of life of Brazilian older adults. Int Nurs Rev 2009; 56:109-15.

17. Min SK, Kim KI, Lee CI, Jung YC, Suh SY, Kim DK. Development of the Korean versions of WHO Quality of Life scale and WHOQOL-BREF. Qual Life Res 2002; 11:593-600.

18. Kalfoss MH, Low G, Molzahn AE. The suitability of the WHOQOL-BREF for Canadian and Norwegian older adults. Eur J Ageing 2008; 5:77-89.

19. Development of the World Health Organization WHOQOL-BREF quality of life assessment. The WHOQOL Group. Psychol Med 1998; 28:551-8.

20. Moreno AB, Faerstein E, Werneck GL, Lopes CS, Chor D. Propriedades psicométricas do Instrumento Abreviado de Avaliação de Qualidade de Vida da Organização Mundial da Saúde no Estudo Pró-Saúde. Cad Saúde Pública 2006; 22:2585-97.

21. Chachamovich E, Fleck MP, Power M. Literacy affected ability to adequately discriminate among categories in multipoint Likert scales. J Clin Epidemiol 2009; 62:37-46.

22. Chung WS, Lan YL, Yang MC. Psychometric testing of the short version of the world health organization quality of life (WHOQOL-BREF) question naire among pulmonary tuberculosis patients in Taiwan. BMC Public Health 2012; 12:630.

23. Jaracz K, Kalfoss M, Gorna K, Baczyk G. Quality of life in Polish respondents: psychometric properties of the Polish WHOQOL-BREF. Scand J Caring Sci 2006; 20:251-60.

24. Li K, Kay NS, Nokkaew N. The performance of the World Health Organization's WHOQOL-BREF in assessing the quality of life of Thai college students. Soc Indic Res 2009; 90:489-501.

25. Skevington SM, Lotfy M, O'Connell KA; WHOQOL Group. The World Health Organization's WHOQOL-BREF quality of life assessment: psychometric properties and results of the international field trial. A report from the WHOQOL group. Qual Life Res 2004; 13:299-310.

26. von Steinbüchel N, Lischetzke T, Gurny M, Eid M. Assessing quality of life in older people: psychometric properties of the WHOQOL-BREF. Eur J Ageing 2006; 3:116-22.

27. Xia P, Li N, Hau KT, Liu C, Lu Y. Quality of life of Chinese urban community residents: a psychometric study of the mainland Chinese version of the WHOQOL-BREF. BMC Med Res Methodol 2012; 12:37.

28. Fang CT, Hsiung PC, Yu CF, Chen MY, Wang JD. Validation of the World Health Organization quality of life instrument in patients with HIV infection. Qual Life Res 2002; 11:753-62.

29. Taylor WJ, Myers J, Simpson RT, McPherson KM, Weatherall M. Quality of life of people with rheumatoid arthritis as measured by the World Health Organization Quality of Life Instrument, short form (WHOQOL-BREF): score distributions and psychometric properties. Arthritis Rheum 2004; 51:350-7. 
30. Trompenaars FJ, Masthoff ED, Van Heck GL, Hodiamont PP, De Vries J. Content validity, construct validity, and reliability of the WHOQOL-BREF in a population of Dutch adult psychiatric outpatients. Qual Life Res 2005; 14:151-60.

31. Usefy AR, Ghassemi GR, Sarrafzadegan N, Mallik S, Baghaei AM, Rabiei K. Psychometric properties of the WHOQOL-BREF in an Iranian adult sample. Community Ment Health J 2010; 46:139-47.

32. Chen K-H, Wu C-H, Yao G. Applicability of the WHOQOL-BREF on early adolescence. Soc Indic Res 2006; 79:215-34.

33. Fleck MP, Louzada S, Xavier M, Chachamovich E, Vieira G, Santos L, et al. Aplicação da versão em português do instrumento abreviado de avaliação da qualidade de vida "WHOQOL-BREF". Rev Saúde Pública 2000; 34:178-83.

34. Hair JF, Black WC, Babin BJ, Anderson RE, Tatham RL. SEM: confirmatory factor analysis. $6^{\text {th }}$ Ed. Porto Alegre: Bookman; 2009.

35. Brown TA. Confirmatory factor analysis for applied research. New York: The Guilford Press; 2006.
36. Muthén BO. Mplus technical appendices. Los An geles: Muthén \& Muthén; 1998.

37. Reichenheim ME, Moraes CL. Operationalizing the cross-cultural adaptation of epidemiological measurement instruments. Rev Saúde Pública 2007; 41:665-73.

38. Division of Mental Health and Prevention of Substance Abuse, World Health Organization. Program on mental health: WHOQOL user manual. Geneva: World Health Organization; 1998.

39. Ministério do Trabalho e Emprego. Classificação brasileira de ocupações: CBO 2002. http://www. mtecbo.gov.br/cbosite/pages/informacoesGerais jsf\#11 (acessado em 17/Set/2011).

Recebido em 14/Dez/2011

Versão final reapresentada em 21/Dez/2012

Aprovado em 27/Fev/2013 